\title{
Interpersonal psychotherapy versus treatment as usual for PTSD and depression among Sichuan earthquake survivors: a randomized clinical trial
}

\author{
Rui Fang Jiang ${ }^{1}$, Hui Qi Tong ${ }^{2}$, Kevin L Delucchi ${ }^{2}$, Thomas C Neylan², Qijia Shi ${ }^{1}$ and Susan M Meffert ${ }^{1 *}$
}

\begin{abstract}
Background: Without effective treatment, PTSD and depression can cause persistent disability in disaster-affected populations.

Methods: Our objective was to test the efficacy of Interpersonal Psychotherapy (IPT) delivered by trained local personnel compared with treatment as usual (TAU) for Posttraumatic Stress Disorder (PTSD) and Major Depressive Disorder (MDD) among adults affected by the Sichuan 2008 earthquake. A small randomized controlled trial of IPT + TAU versus TAU alone was delivered by local mental health personnel in Shifang, China. Between July 2011 and January 2012, 49 adults $\geq 18$ years with PTSD, MDD or both were enrolled and randomized to 12 weekly sessions of IPT + TAU (27) or TAU (22) alone $\times 12$ weeks. IPT was then offered to the TAU group. Unblinded follow up assessments were conducted at three and six months. IPT was a 12 session, weekly one hour treatment delivered by local personnel who were trained and supervised in IPT. TAU was continuation of prescribed psychotropic medication (if applicable) and crisis counseling, as needed.

Main Outcome(s) and Measures (s): Clinician Administered PTSD Scale (CAPS) PTSD diagnosis; Structured Clinical Interview for DSM-IV (SCID) for MDD diagnosis. Secondary measures included PTSD/depression symptoms, interpersonal conflict/anger, social support, self-efficacy and functioning.
\end{abstract}

Results: Using an intent-to-treat analysis, 22 IPT + TAU and 19 TAU participants were compared at three months post-baseline. A significantly greater reduction of PTSD and MDD diagnoses was found in the IPT group (51.9\%, 30.1\%, respectively) versus the TAU group (3.4\%, 3.4\%, respectively). Despite the small sample, the estimates for time-by-condition analyses of target outcomes ( 2.37 for PTSD $(p=.018)$ and 1.91 for MDD $(p=.056)$ ) indicate the improvement was better in the IPT + TAU condition versus the TAU group. Treatment gains were maintained at 6 months for the IPT group. A similar treatment response was observed in the TAU group upon receipt of IPT.

Conclusions: This initial study shows that IPT is a promising treatment for reducing PTSD and depression, the two major mental health disorders affecting populations surviving natural disaster, using a design that builds local mental health care capacity.

Trial Registration: ClinicalTrials.Gov number, NCT01624935.

Keywords: Earthquake, Disaster, Mental health, Posttraumatic stress disorder, Depression, Domestic violence

\footnotetext{
* Correspondence: Susan.Meffert@ucsf.edu

'Wuhan Hospital for Psychotherapy, Wuhan Mental Health Center, Tongji Medical College Huazhong University of Science and Technology, Kaiming Road 44\#, Wuhan 430019, China

Full list of author information is available at the end of the article
} 


\section{Background and Rationale}

Seventy-five percent of people living with serious mental illness in Low and Middle Income Countries (LMICs) never receive treatment [1]. When natural disaster occurs in LMICs, additional mental health care needs emerge. The 2008 Sichuan earthquake resulted in the deaths of 69,179 people, including more than 5,000 students in school at the time of the event. PTSD and depression were estimated to affect $65 \%$ of the surviving population [2-6].

We conducted a mental health care needs assessment (2009-2010) in collaboration with the Wuhan Hospital for Psychotherapy (WHP), an early mental health care responder, that included interviews and focus groups with survivors, healthcare providers and community leaders. We found that depression, PTSD and relationship distress were significant problems and were commonly ascribed to interpersonal loss, spousal conflict after children's deaths, and transitions related to material and job loss. Individual treatment (versus group) was desired. Although psychotherapies such as exposure therapy and cognitive processing therapy are first line treatments for trauma-related disorders in Europe and North America, and have been used successfully in LMICs [7], our mental health care needs assessment in this disaster-affected Sichuan population indicated that PTSD symptoms were one component of broader emotional distress and pervasive disruption of relationships. Drawing on prior studies showing the efficacy of IPT in LMICs and with trauma-affected populations [8-11], we theorized that the relational focus of Interpersonal Psychotherapy (IPT) would be well-aligned with local needs. IPT delivered by area personnel was selected in order to contribute to capacity building.

IPT is a 12-week structured psychotherapy developed by Klerman and Weissman in the 1980s and an established first line treatment for depression in the U.S. $[12,13]$. The goal of IPT is to examine and change current relationships and social support in order to improve mood and anxiety symptoms. IPT has been modified for other uses, including delivery by trained paraprofessionals in culturally distinct settings [8]. To our knowledge, this is the first study of IPT for disaster-related mental disorders in LMICs and the first to use eligibility criteria that allow for PTSD and/ or Major Depressive Disorder (MDD) - two major mental health sequelae of traumatic stress [14].

\section{Objectives and Hypotheses}

This article reports the results of a pilot randomized controlled clinical trial of IPT plus treatment as usual (TAU) versus TAU alone for Sichuan earthquake survivors, using a capacity building design with permissive eligibility criteria. The study took place between July 2011 and April 2012. Our purpose was to pilot test the efficacy of IPT for PTSD and MDD (primary outcomes), interpersonal factors and functioning. We hypothesized that IPT + TAU would reduce diagnosis and symptoms of PTSD and MDD, reduce interpersonal conflict and improve social support and functioning, relative to TAU, alone.

\section{Methods \\ Participants}

Participants were 18 years of age or older, able to attend weekly sessions, give verbal informed consent, met criteria for PTSD as assessed by the CAPS and/or MDD, as assessed by the SCID. Exclusion criteria were psychosis, cognitive dysfunction or substance abuse/dependence requiring a different type of care. Participants taking prescribed psychotropic medication, including Selective Serotonin Reuptake Inhibitors (SSRIs), Selective Serotonin and Norepinephrine Reuptake Inhibitors (SNRIs) and benzodiazepines, were eligible for the study.

Informed by our prior mental health care needs assessment, recruitment efforts focused on locations with high concentrations of individuals who continued to suffer mental health effects from the 2008 earthquake despite the intervening time. Two of these were mental health care centers (Shifang Counseling Center and Shifang People's Hospital) and the third was a high school that had been heavily affected by the earthquake. Participants were referred to the study by mental health care providers and recruited from the high school through a general screening. No siblings, parents or teachers of study participants were included. Prospective participants provided informed consent prior to screening for study eligibility.

\section{Procedures}

This was a two-group parallel, randomized trial of IPT + TAU versus TAU in Sichuan, China. Following the 12 week IPT + TAU, those assigned to TAU were offered IPT treatment. All study procedures were approved by the University of California San Francisco Committee on Human Research and its counterpart at WHP. Verbal consent was used because of incomplete literacy.

Given that this was a small pilot study of IPT for disaster survivors, sample size was not based on hypothesis testing but on issues of feasibility within budget constraints. We estimated that at least 20 individuals per arm would be needed to detect a clinically relevant treatment effect $[15,16]$. Individuals were randomized to IPT + TAU or TAU in a 1:1 fashion, stratified by gender using blocks of size six to ensure approximate balance.

Seven mental health professionals from WHP completed the participants' consents, screening and baseline assessments. In the original plan, participants were assessed by personnel blinded to subject group assignment at three and six month time-points. Unexpected personnel and 
budget constraints required that the un-blinded study coordinator and un-blinded assistant carry out these assessments. The study coordinator and study assistant each conducted approximately half of the assessments. They worked independently and did not assess the same group of participants at each time-point.

\section{Treatments}

\section{Treatment as usual (TAU)}

TAU included continuation of SSRIs, SNRIs, benzodiazepines and crisis counseling services. Those receiving medication met weekly with psychiatrists for medication management and had access to mental health professionals for interim crisis care. For those not on medication, TAU consisted of mental health crisis services, as needed.

\section{IPT + TAU}

IPT Adaptation. Traditional IPT was modified slightly to address trauma-related mental disorders of the local population. IPT focuses on one of four areas, depending on the etiology of the patient's distress -interpersonal disputes, role transitions, grief/loss or interpersonal sensitivity/deficit [17]. For the purposes of this study, the last category was eliminated, as it relates to lifelong patterns of relational deficits often driven by character traits, whereas the focus of this study was on testing IPT for trauma-related mental disorders.

IPT was delivered in one hour weekly individual sessions for 12 weeks. IPT participants also received TAU. For participants taking medication, IPT and medication management were delivered by the same clinician in the same weekly session.

Prospective study therapists were recruited through our collaborators and intensively trained in IPT for two weeks in June of 2011 by S Meffert. Those who completed the course and successfully modeled a beginning, middle and end session of IPT were invited to join the study as therapists-six women and four men. Five were psychologists working at Shifang Counseling Center, four were psychiatrists working at Shifang People's Hospital, and one was a teacher experienced with processing emotional trauma secondary to the earthquake. Therapists were assigned one practice IPT case and study cases were added to caseloads as practice IPT sessions were successfully accomplished. IPT was supervised by an onsite psychologiststudy coordinator (RF Jiang) or remotely by a psychologist located in San Francisco and originally from China (HQ Tong). Both were experienced with IPT delivery and spoke weekly with S Meffert to monitor therapists' application of IPT and address any questions.

\section{IPT Adherence}

Treatment fidelity was assessed for each session by the therapist supervisor. Supervisors rated therapists' adherence to IPT protocol using a ten-point scale assessing overall quality of the session (three items) and quality of key components for each of four phases (2-5 items), and two reverse coded items for off-protocol treatments, such as CBT.

All primary and secondary measures were administered by research psychologists from WHP at each timepoint (baseline, three and six months).

\section{Primary outcomes}

Primary outcomes were diagnosis of PTSD on the CAPS and/or diagnosis of MDD on the SCID, both of which are widely used in China $[4,18,19]$. We are not aware of psychometric assessments (reliability or validity) focusing specifically on the use of the SCID/CAPS with a Chinese population.

\section{Secondary outcomes}

A standard process of forward/backward translation with resolution of discrepancies was used [20-22]. Two mental health professionals from WHP, bilingual in English and the local Mandarian dialect and experienced with emotional distress in the local population, separately translated English versions into local Mandarian. Discrepancies were resolved through discussion and consensus. Two additional personnel then separately back-translated the measures from Mandarian into English, compared and resolved discrepancies. The Cronbach alphas observed in this sample were calculated using Proc Corr in SAS v9.3.

Beck Depression Inventory (BDI-II) is a widely used 21item depression symptom measure [23]. It has shown strong convergent validity with other measures of depression and has a Cronbach's alpha of .91 for outpatient populations [24]. The psychometric properties of the Chinese BDI have been tested with other populations in China, demonstrating strong reliability with Cronbach's alpha of .85 , and strong construct validity with the exception of the item addressing loss of libido [25]. Cronbach's alpha in this sample was 92 .

Generalized Self-Efficacy (GSE) is a 10-item psychometric scale designed to assess optimistic self-beliefs [26]. Across different language versions, the GSE Cronbach's alpha ranges from .75 to .91 and has convergent validity with other social cognitive variables [27]. Cronbach's alpha in this sample was .86 .

State Trait Anger (STAXI) is a widely used measure of state and trait anger consisting of 15 and 10 items, respectively. Cronbach's alpha has been shown to be .93 and .86 for state and trait scales, respectively [28]. Construct (convergent) validity is supported by its high correlation with the Buss-Drukee and Cook-Medley scales. A Chinese version of the STAXI has been tested with two samples of Hong Kong populations, which showed supported construct validity and reliability [29]. The Cronbach's alphas for this sample were .93 (STAXIstate) and .85 (STAXI-trait). 
Conflict Tactics Scale (CTS) is a 10-item widely used scale for identifying intimate partner maltreatment. For the purposes of this study, we used the CTS subscales corresponding to IPV, including total couple violence, respondent's victimization and perpetration. Cronbach's alphas for the scales are $.88, .83$ and .82 , respectively. Construct validity of the CTS is supported by correlation with other measures of aggression [30]. Cronbach's alpha for this sample was 90 .

Social Adjustment Scale (SAS) is a 54-item scale measuring role performance. The SAS has established construct and criterion validity and Cronbach's alpha of .74 [31]. In this sample, the SAS social/leisure subscale had a Cronbach's alpha of 82 .

Quality of Life Index (QLI) is a widely used 24-item scale that measures well-being. The QLI has established construct and convergent validity with a Cronbach's alpha of .93 for the overall scale and .87 for the health and functioning subscale [32]. In this sample, the overall scale and the health/functioning subscale had Cronbach's alphas of .95 and .73 , respectively.

\section{Statistical analysis}

Data was described using standard summary statistics. The main analysis consisted of comparing the change from baseline to post-test (three months) between the two treatment conditions. The two primary outcome measures of PTSD and MDD diagnosis were analyzed by estimating and testing a statistical model of each dichotomous measure with a binomial distribution and logit link using Generalized Estimating Equations to account for clustering of participants by therapist (therapist effect) and the repeated assessments. An unstructured covariance matrix was specified. Models included terms for time (baseline and post-test), treatment condition, their interaction and the participant's gender. Parallel models of the secondary scales were also tested using a continuous distribution in place of the binomial. For these tests of treatment effect, all available data was used to estimate and test the statistical models.

Given the limited sample size and the focus on end-oftreatment effects, the percent in each condition diagnosed with PTSD or MDD and their associated symptom levels were tested for significant change from the three-month post-test to the six-month follow-up within each of the conditions using McNemar's test and repeated measures t-tests. All analyses were conducted under the intent-totreat principle and all available data was used on testing. Analysis was conducted using SAS v9.3.

\section{Results}

A total of 219 participants were screened AugustNovember 2011 (Figure 1). 207 of these were part of a general screening of all students attending a high school with heavy earthquake exposure, of which 38 met study criteria and 37 agreed to participate (18\%). Twelve participants were referred from mental health clinics (Shifang Counseling Center and Shifang People's Hospital) - all met study eligibility criteria and all agreed to participate. In total, of the 50 eligible study participants, 49 (98\%) agreed to participate.

All study participants completed baseline measures. Twenty-seven participants were assigned to IPT + TAU. Of the 22 individuals who began IPT, 19 completed two or more sessions (86\%) and 16 completed all 12 sessions (73\%). Five of the IPT + TAU group were lost to followup between baseline and the three-month assessment, including one who moved away from the area. Of the 22 participants assigned to TAU, 19 participants completed the three-month assessment. Sixteen of the TAU participants began IPT when it was offered at three months; $100 \%$ of these completed two or more sessions and 93\% completed all 12 sessions (Figure 1). One participant assigned to a psychologist was identified as having symptoms of psychosis prior to starting IPT and was referred to a psychiatric inpatient unit for hospitalization and medication. None of the other participants in either group required crisis counseling, medication changes/ initiation or psychiatric consultation during the study.

Baseline sample characteristics of the participants are shown in Table 1. $18.5 \%$ of the IPT + TAU group was taking SSRI/SNRI and benzodiazepine, 3.7\% was taking only SSRI/SNRI ( $22 \%$ total taking medication). $9.1 \%$ of the TAU group was taking SSRI/SNRI and benzodiazepine and $9.1 \%$ for was taking only SSRI/SNRI (18\% total taking medication). The majority of study participants were women and most identified the 2008 earthquake as their most stressful life event.

At baseline, $45.5 \%$ of the TAU and $66.7 \%$ of the IPT + TAU participants were positive for PTSD (Table 2). At the post-test, $42.1 \%$ in the TAU and $13.6 \%$ of the IPT + TAU condition had PTSD. GEE parameter estimates were -2.58 for time ( $\mathrm{p}=.001),-1.52$ for condition $(\mathrm{p}=.055)$ and 2.37 for time by condition $(\mathrm{p}=.018)$. At baseline, $59.1 \%$ of the TAU and $51.9 \%$ of the IPT + TAU participants were positive for MDD (Table 2). At the post-test, $57.9 \%$ in the TAU and $18.2 \%$ of the IPT + TAU condition were positive. GEE parameter estimates were -1.75 for time $(\mathrm{p}=.023),-1.99$ for condition $(\mathrm{p}=.007)$ and 1.94 for time by condition $(\mathrm{p}=.056)$. Secondary outcome baseline scores were similar between groups (Table 3). For those participants in the IPT + TAU group who had MDD at baseline, 92\% had remitted and no longer met criteria for MDD following treatment (three months). IPT + TAU participants with PTSD at baseline had a remission rate of $58 \%$ at three months. In comparison, at three months, TAU recipients had remission rates of $30 \%$ and $0 \%$ for MDD and PTSD, respectively. At the six-month follow up, the IPT + TAU 


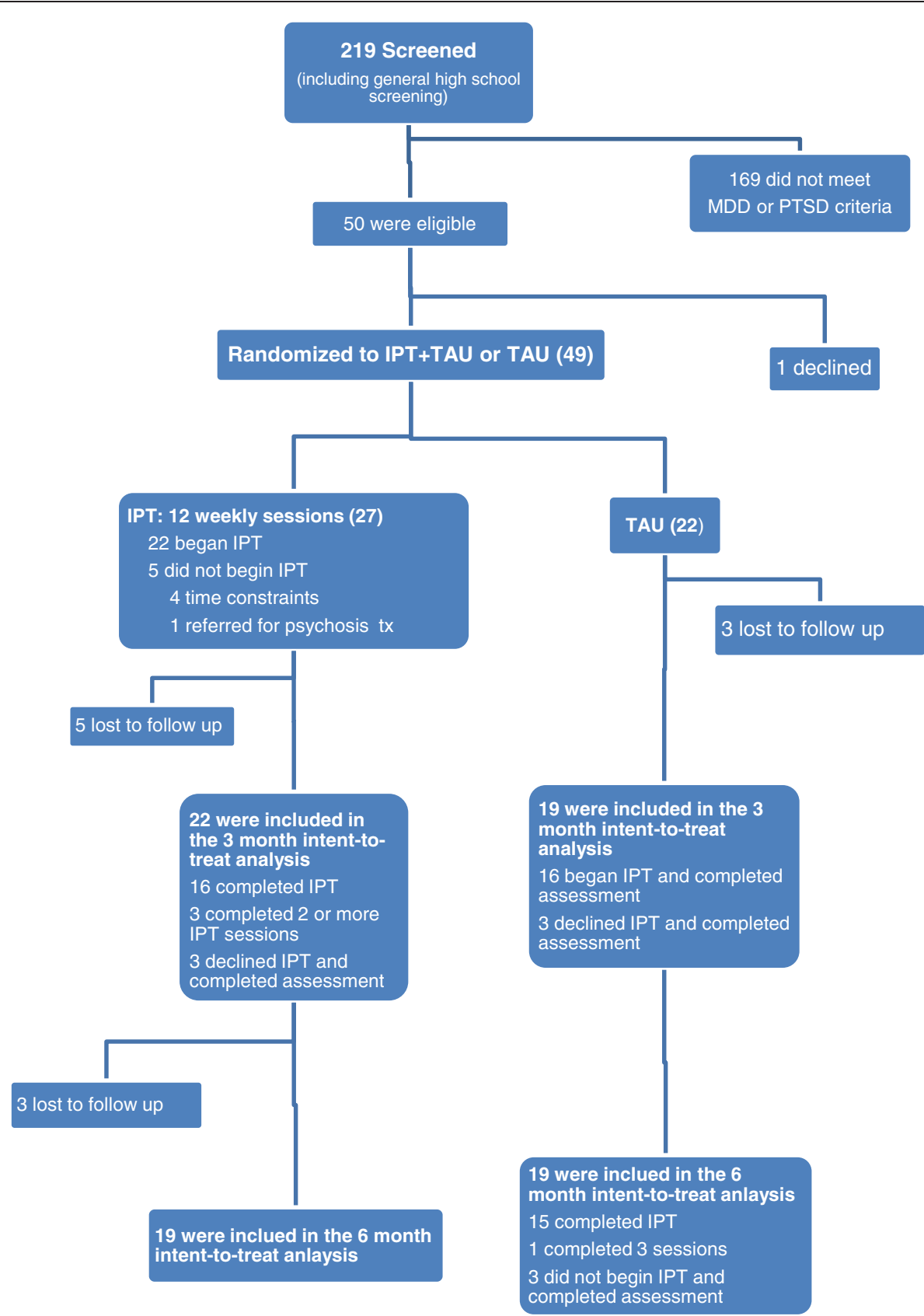

Figure 1 Flow of participants through study. IPT: Interpersonal Psychotherapy; MDD: Major Depressive Disorder; PTSD: Posttraumatic Stress Disorder; TAU: Treatment As Usual; Tx: Treatment.

group maintained treatment gains and had further reduction of PTSD and MDD diagnoses. Upon receipt of IPT, the TAU group demonstrated reductions in PTSD and MDD diagnoses similar to that observed in the IPT group. Neither therapist nor supervisor assignment had an effect on IPT outcomes.

Analysis of PTSD and depression symptoms (as opposed to diagnoses) also found a strong IPT effect (Figure 2). Cohen's d effect sizes for PTSD symptoms on CAPS and depression symptoms on the BDI were 1.01 and .79 , respectively (Table 3). Significant effects were found on all three models terms of time, condition and the interaction for CAPS symptoms and BDI symptoms. We conducted exploratory analyses of the effects of gender and medication use on IPT response. Gender had no effect on IPT response for PTSD or MDD diagnosis or symptoms. Use of antidepressant (SSRI or SNRI) or benzodiazepines did not predict diagnosis with PTSD or MDD, however, it was 
Table 1 Baseline characteristics of study sample

\begin{tabular}{lll}
\hline Variable & $\begin{array}{l}\text { IPT + TAU }(\mathbf{n}=\mathbf{2 7}) \\
\text { Mean/number } \\
\text { (SD or\%) }\end{array}$ & $\begin{array}{l}\text { TAU }(\mathbf{n}=\mathbf{2 2}) \\
\text { Mean/number } \\
\text { (SD or\%) }\end{array}$ \\
\hline Age & $24.79(11.66)$ & $36.05(15.68)$ \\
Women & $18(62)$ & $17(77)$ \\
Medication (total) & $6(22)$ & $4(18)$ \\
$\quad$ SSRI/SNRI + BZ & $5(18.5)$ & $2(9.1)$ \\
$\quad 1(3.7)$ & $2(9.1)$ \\
SSRI/SNRI & & \\
Most traumatic life event & $21(72)$ & $20(77)$ \\
Earthquake & $2(7)$ & 0 \\
Interpersonal & $4(15)$ & $2(9)$ \\
Other &
\end{tabular}

BZ: benzodiazepine; IPT: Interpersonal Psychotherapy; SD: Standard Deviation; SNRI: Selective Serotonin and Norepinephrine Reuptake Inhibitor; SSRI:

Selective Serotonin Reuptake Inhibitor; TAU: Treatment as Usual.

associated with higher PTSD and depression symptoms. IPT remained a significant predictor of diagnosis and symptom response even in the presence of antidepressant and benzodiazepine use.

Although the study was not designed to detect changes in anger and interpersonal violence, IPT had moderatelarge effects on anger and violent victimization by an intimate partner (Table 3). The term for condition was significant for trait anger and just missed the cutoff for state anger ( $\mathrm{p}=.053)$. IPT also had a large effect on increasing social adjustment on the SAS social/leisure subscale. The social/leisure subscale was significant for time, condition and interaction, as were the student and parental subscales when applicable to the participant. IPT had a large effect on increasing self-efficacy, quality of life total score and the health and function subscale. Significant effects were found on all three models terms of time, condition and the interaction for self-efficacy. The quality of life total score and health and function subscale showed significant change over time and condition.

Mean IPT adherence was 8.0 with a range of 7.3 to 9.7. We observed a graduated response for MDD such that greater adherence to IPT protocol was significantly and inversely associated with MDD diagnosis at the conclusion of treatment.

\section{Discussion}

IPT delivered by local personnel was effective for reducing chronic PTSD and depression symptoms, as well as full diagnosis of PTSD and MDD among Sichuan earthquake survivors. IPT increased overall quality of life, social support and self-efficacy, while reducing anger and receipt of violent victimization. Treatment gains were maintained three months following completion of the intervention with further spontaneous improvement, although additional follow up is necessary to assess maintenance of treatment gains. Similar reductions in PTSD and depression were observed for TAU participants after they received IPT.

Although this study was not designed to detect the relational effects of IPT, we found lowered rates of intimate partner violence (IPV) toward IPT + TAU recipients, consistent with a psychotherapy trial of IPV survivors in the U.S. which found that reduction of PTSD and depression was associated with lower risk of future IPV [33]. Iverson and colleagues assessed the effect of CBT for depression and PTSD among female survivors of interpersonal violence on IPV in the six months following the study $(n=150)$. Reductions in PTSD and depression were associated with decreased risk of IPV in the six months following the study, controlling for recent IPV in a current relationship. Treatment response correlated with lower IPV levels at the six-month follow-up, such that individuals who experienced a greater reduction of PTSD and/or depression symptoms reported lower IPV levels at six-month follow-up compared with those who had poorer treatment response. Although the mechanism is not yet clear, Iverson and colleagues drew on PTSD literature, positing that numbing symptoms may put women at risk of IPV by impairing their ability to detect and respond to danger cues. Depression was theorized to affect victimization by decreasing cognitive

Table 2 Primary outcomes: baseline and 3 months by group

\begin{tabular}{|c|c|c|c|c|c|}
\hline \multirow{2}{*}{$\begin{array}{l}\text { Variable } \\
\text { Baseline }\end{array}$} & \multicolumn{2}{|c|}{ IPT + TAU $(n=27)$} & \multicolumn{2}{|c|}{ TAU $(n=22)$} & \multirow[t]{2}{*}{ Chi-Square } \\
\hline & No. & $\%$ & No. & $\%$ & \\
\hline PTSD diagnosis (CAPS) & 18 & 66.7 & 10 & 45.5 & 2.05 \\
\hline Depression diagnosis (SCID) & 14 & 51.9 & 12 & 59.1 & .04 \\
\hline 3 Months & \multicolumn{2}{|c|}{ Post-IPT $(n=22)$} & \multicolumn{2}{|c|}{ Post-TAU $(n=19)$} & \\
\hline PTSD diagnosis (CAPS) & 3 & 13.6 & 8 & 42.1 & 4.21 \\
\hline Depression diagnosis (SCID) & 4 & 18.2 & 11 & 57.9 & $6.93^{*}$ \\
\hline
\end{tabular}

${ }^{*} \mathrm{p} \leq .01$.

CAPS: Clinician Administered Posttraumatic Stress Disorder (PTSD) Scale; IPT: Interpersonal Psychotherapy; PTSD: Posttraumatic Stress Disorder; SCID: Structured Clinical Interview for DSM-IV; SD: Standard Deviation; SNRI: Selective Serotonin and Norepinephrine Reuptake Inhibitor; SSRI: Selective Serotonin Reuptake Inhibitor; TAU: Treatment as Usual. 
Table 3 Secondary outcomes: baseline and 3 months by group

\begin{tabular}{|c|c|c|c|c|c|}
\hline \multirow[b]{2}{*}{ Baseline } & \multicolumn{2}{|c|}{ IPT + TAU $(n=29)$} & \multicolumn{2}{|c|}{ TAU $(n=22)$} & \\
\hline & No. or mean & SD or $\%$ & No. or mean & SD or $\%$ & \\
\hline PTSD symptoms (CAPS) & 39.41 & 15.38 & 45.05 & 11.06 & \\
\hline Depression symptoms (BDI) & 20.7 & 11.6 & 21.8 & 12.7 & \\
\hline Anger (state) & 23.83 & 8.61 & 26.38 & 11.34 & \\
\hline Anger (trait) & 19.45 & 5.43 & 20.86 & 5.24 & \\
\hline Quality of life index(QLI) & 17.12 & 5.07 & 14.07 & 3.73 & \\
\hline QLI Health and function subscale & 15.93 & 5.90 & 13.03 & 3.82 & \\
\hline Social support (SAS) & 2.93 & .79 & 3.05 & .67 & \\
\hline \multicolumn{6}{|l|}{ Social/leisure subscale } \\
\hline Interpersonal conflict (CTS) & 1.15 & 2.92 & 2.10 & 6.51 & \\
\hline \multicolumn{6}{|l|}{ Partner violence subscale } \\
\hline \multirow[t]{2}{*}{ Self-Efficacy } & 24 & .90 & 24.18 & .90 & \\
\hline & \multicolumn{2}{|c|}{ IPT + TAU $(n=19)$} & \multicolumn{2}{|c|}{$\operatorname{TAU}(n=19)$} & \\
\hline 3 Months & No. or mean & SD or $\%$ & No. or mean & SD or $\%$ & Cohen $\mathrm{d}$ (row) \\
\hline PTSD symptoms (CAPS) & 19.59 & 17.94 & 38.74 & 19.76 & -1.01 \\
\hline Depression symptoms (BDI) & 10.6 & 13.2 & 20.7 & 12.5 & -.79 \\
\hline Anger (state) & 17.8 & 5.7 & 25.58 & 10.78 & -.90 \\
\hline Anger (trait) & 17.00 & 4.77 & 20.37 & 5.28 & -.67 \\
\hline Quality of life index(QLI) & 19.90 & 6.07 & 15.07 & 4.62 & .90 \\
\hline QLI Health and function subscale & 19.40 & 6.98 & 14.07 & 4.60 & .90 \\
\hline Social support (SAS) & 2.47 & .70 & 3.03 & .61 & -.85 \\
\hline \multicolumn{6}{|l|}{ Social/leisure subscale } \\
\hline Interpersonal conflict (CTS) & .18 & .50 & 1.74 & 5.77 & -.38 \\
\hline Partner violence subscale & & & & & \\
\hline
\end{tabular}

BDI: Beck Depression Inventory; CAPS: Clinician Administered Posttraumatic Stress Disorder (PTSD) Scale; CTS: Conflict Tactics Scale; IPT: Interpersonal Psychotherapy; PTSD: Posttraumatic Stress Disorder; QLI: Quality of Life Index; SAS: Social Adjustment Scale; SD: Standard Deviation; TAU: Treatment as Usual.

and affective capacity, as well as self-worth. Clearly, neither the Iverson study nor the secondary findings presented here suggest that IPV is in any way brought on by the survivor, but rather that improving the mental health of an IPV survivor may provide them with better access to their full emotional and cognitive capacities in order to grapple with the threat of future IPV. Of course, mental health treatment of IPV survivors could never supplant strenuous efforts halt perpetration; rather such treatments might augment larger societal efforts to change gender norms and reduce IPV.

IPT + TAU participants who were parents reported improvement in the quantity and quality of interactions with children. Although the numbers are small, these findings together with observed improvements in social support are encouraging in regards to IPT's potential contribution to social reconstruction in traumatized communities. In a post-disaster setting with highly disrupted social structures, it is possible that an interpersonally focused treatment such as IPT may both improve symptoms of depression and PTSD, and reconstitute social support, a central factor in recovery from traumatic stress [34-36]. However, it is important to note that this study took place two years after the earthquake and findings may not be generalizable to the immediate disaster response.

This study used a brief training period, followed by onsite or remote supervision. The success of remote supervision with minimal ground support is promising, given the need for global mental health care scale up.

In this study, the cost of one PTSD and/or MDD cure was approximately 200USD for therapist payment (total cures/therapist payments) and 177USD for therapist supervision (total cures/therapist supervisor payments) the latter is expected to decrease as therapists gain experience. For comparison, cure of drug-sensitive TB is $~ 250$ USD and one year of HIV treatment in subSaharan Africa averages 900USD [37-39].

\section{Limitations}

The primary limitation of this study is its size and follow up. As a pilot study, the goal was to determine the efficacy of IPT for common mental health sequelae following the 


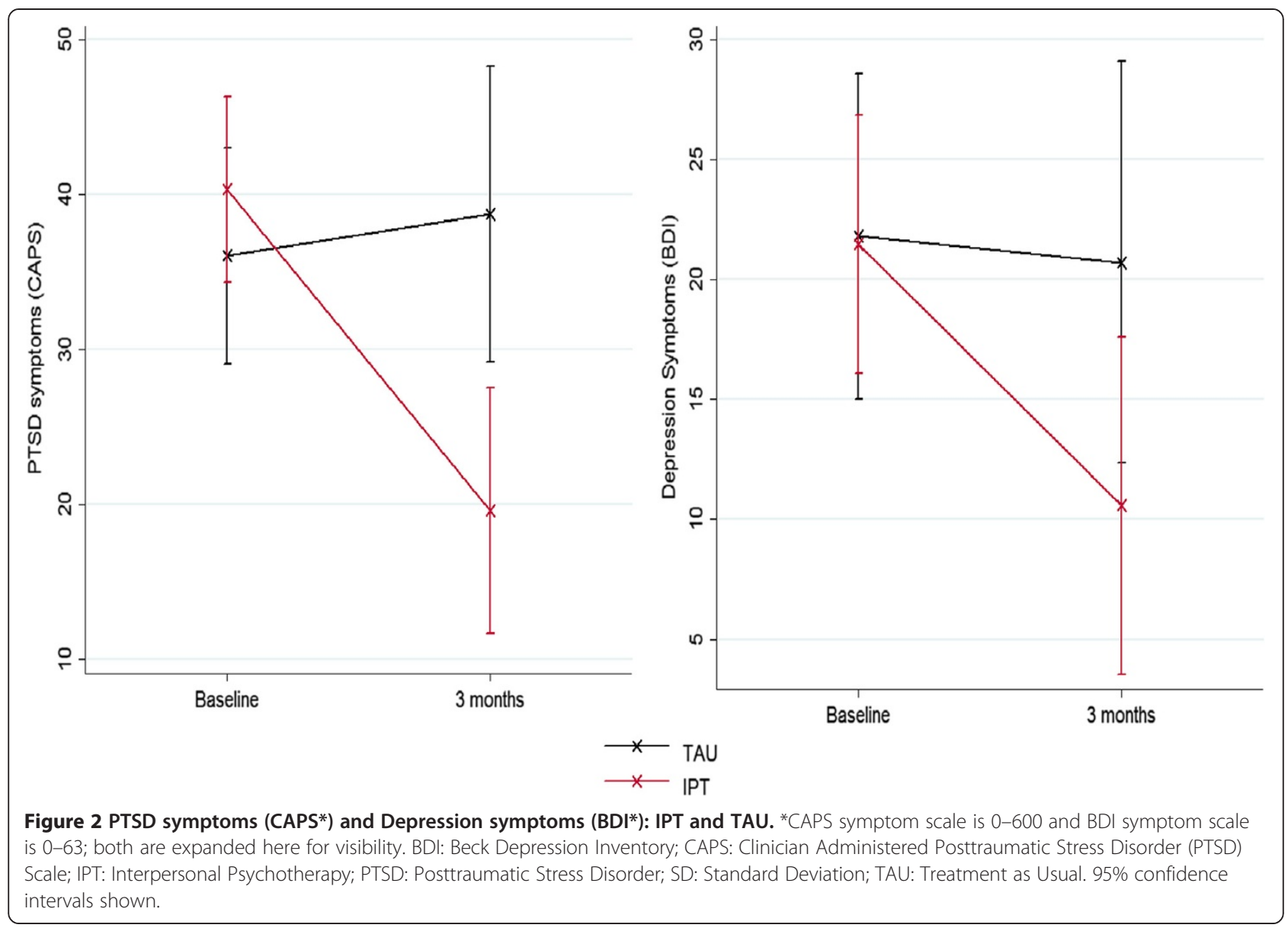

Sichuan earthquake. Larger size and longer follow up were limited by financial constraints.

A second limitation of this study is the lack of blinding to treatment status, which could introduce bias in favor of IPT efficacy. The possibility of bias may be mitigated by the fact that both symptom and diagnostic measures showed similar patterns of IPT response, and that spontaneous remissions were observed in the TAU and the IPT + TAU group in nontreatment phases.

Given the length of time between the earthquake and the start of this study, most participants had chronic psychopathology. It is encouraging that IPT was effective for chronic PTSD/MDD, but findings may not be generalizable to populations more recently affected.

The use of TAU, rather than a therapy control designed to meet with the same frequency and duration of IPT, limits the interpretation of the data in regards to the mechanism of change fostered by IPT.

Finally, given the size of the samples required to conduct psychometric analyses, the measures used in this study were not validated with the local Sichuan population. Although widespread use of CAPS and SCID in
China $[18,19,40]$ does not guarantee validity, the symptom measures, including the BDI and the PTSD Checklist (PCL) (latter not reported here), showed a pattern of results similar to those observed with diagnostic measures, suggesting convergent validity.

\section{Conclusions}

This study contributes to the field of global mental health research by providing data on use of IPT in China and first use with survivors of natural disaster. This and other studies [7-9,15,41,42] now constitute a sizable body of research showing efficacy for culturally-adapted IPT and other evidence-based psychotherapies delivered in a feasible and effective manner by local personnel for common mental disorders in LMICs. Given the pressing need to find sustainable treatments to address the mental health care gap [43-45], the accumulation of this data is exciting. Larger studies with long-term follow up and active controls, as well as cost-effectiveness measures are next steps for global mental health intervention research with traumatized populations. 


\section{Competing interests}

The authors declare that they have no competing interests.

\section{Authors' contributions}

RJ supervised IPT, carried out data collection, compiled data for analysis and assisted with drafting the manuscript. HT supervised IPT, assisted with data collection and assisted with drafting the manuscript. KD conceptualized and performed the data analysis. TN and QS assisted with study design and execution. SM conceived of the study, participated in its design, execution, data analysis and drafted the manuscript. All authors read and approved the final manuscript.

\section{Acknowledgements}

We would like to thank the Wuhan Hospital for Psychotherapy for their support in this endeavor. We would like to thank Dr. Du of the Shifang People's Hospital and Mr. Xiao of the Shifang Counseling Center for both of their teams' enthusiasm and hard work on this project. We would like to thank Charles Marmar, MD, Professor and Chair of the Department of Psychiatry at New York University Langone Medical Center, for his assistance with this collaboration. This research was funded by the following awards to S. Meffert: the UCSF Burke Family Global Health Junior Faculty Award, the UCSF Building Interdisciplinary Research Careers in Women's Health (BIRCWH) KL2 award and the UCSF Research Evaluation and Allocation Committee (REAC) Grant: Pilot for Junior Investigators in Basic and Clinical/ Translational Sciences. Authors report no conflicts of interest. RFJ, KD and SM had full access to all the data in the study and take responsibility for the integrity of the data and the accuracy of the data analysis.

\section{Author details}

'Wuhan Hospital for Psychotherapy, Wuhan Mental Health Center, Tongji Medical College Huazhong University of Science and Technology, Kaiming Road 44\#, Wuhan 430019, China. ${ }^{2}$ Department of Psychiatry, University of California, San Francisco, California, USA.

Received: 5 December 2013 Accepted: 17 July 2014

Published: 4 September 2014

\section{References}

1. WHO: WHO Mental Health Gap Action Programme (mhGAP). [http://www.who.int/mental_health/mhgap/en/]

2. Kun $P$, Chen $X$, Han S, Gong X, Chen M, Zhang W, Yao L: Prevalence of post-traumatic stress disorder in Sichuan Province, China after the 2008 Wenchuan earthquake. Public Health 2009, 123:703-707.

3. Kun P, Han S, Chen X, Yao L: Prevalence and risk factors for posttraumatic stress disorder: a cross-sectional study among survivors of the Wenchuan 2008 earthquake in China. Depress Anxiety 2009, 26:1134-1140.

4. Cheng Z, Ma N, Yang L, Agho K, Stevens G, Raphael B, Cui L, Liu Y, Yan B, Ma H, Yu X: Depression and Posttraumatic Stress Disorder in Temporary Settlement Residents 1 Year After the Sichuan Earthquake. Asia Pac J Public Health 2013, Advance Online Publication.

5. Chan CLW, Wang C-W, Ho AHY, Qu Z-Y, Wang X-Y, Ran M-S, Mao W-J, Lu BQ-B, Zhang BQ, Zhang X-L: Symptoms of posttraumatic stress disorder and depression among bereaved and non-bereaved survivors following the 2008 Sichuan earthquake. J Anxiety Disord 2012, 26:673-679.

6. Chan CLW, Wang C-W, Qu Z, Lu BQ, Ran M-S, Ho AHY, Yuan Y, Zhang BQ, Wang $X$, Zhang $X$ : Posttraumatic stress disorder symptoms among adult survivors of the 2008 Sichuan earthquake in China. J Trauma Stress 2011 24:295-302.

7. Bass JK, Annan J, Mclvor Murray S, Kaysen D, Griffiths S, Cetinoglu T, Wachter K, Murray LK, Bolton PA: Controlled trial of psychotherapy for congolese survivors of sexual violence. N Engl J Med 2013, 368:2182-2191.

8. Bolton P, Bass J, Neugebauer R, Verdeli H, Clougherty KF, Wickramaratne P, Speelman L, Ndogoni L, Weissman M: Group interpersonal psychotherapy for depression in rural Uganda: a randomized controlled trial. JAMA 2003, 289:3117-3124.

9. Bolton P, Bass J, Betancourt T, Speelman L, Onyango G, Clougherty KF, Neugebauer R, Murray L, Verdeli H: Interventions for depression symptoms among adolescent survivors of war and displacement in northern Uganda: a randomized controlled trial. JAMA 2007, 298:519-527.
10. Krupnick JL, Green BL, Stockton P, Miranda J, Krause E, Mete M: Group interpersonal psychotherapy for low-income women with posttraumatic stress disorder. Psychother Res 2008, 18:497-507.

11. Campanini RFB, Schoedl AF, Pupo MC, Costa ACH, Krupnick JL, Mello MF: Efficacy of interpersonal therapy-group format adapted to post-traumatic stress disorder: an open-label add-on trial. Depress Anxiety 2010, 27:72-77.

12. Cuijpers P, Van vStraten A, Andersson G, Van Oppen P: Psychotherapy for depression in adults: a meta-analysis of comparative outcome studies. J Consult Clin Psychol 2008, 76:909-922.

13. De Mello MF, De Jesus MJ, Bacaltchuk J, Verdeli H, Neugebauer R: A systematic review of research findings on the efficacy of interpersonal therapy for depressive disorders. Eur Arch Psychiatry Clin Neurosci 2005, 255:75-82.

14. North CS, Pfefferbaum B: Mental health response to community disasters: a systematic review. JAMA 2013, 310:507-518.

15. Meffert SM, Abdo AO, Alla OAA, Elmakki YOM, Omer AA, Yousif S, Metzler TJ, Marmar CR: Sudanese Refugees in Cairo, Egypt: A randomized controlled trial of interpersonal psychotherapy for trauma, depression and interpersonal violence. Psychol Trauma Theory Res Pract Policy 2009, 24:1835-1848.

16. Meffert SM, Ekblad S: Global Mental Health Intervention Research and Mass Trauma. Open Source J Clin Trials in press.

17. Stuart S: Interpersonal therapy: a guide to the basics. Psychiatr Ann 2006, 36:542-550

18. Chen $\mathrm{G}$, Shen $\mathrm{H}$, Chen $\mathrm{G}$ : A cross-sectional study on posttraumatic stress disorder among elderly Qiang citizens 3 years after the Wenchuan earthquake in China. Can J Psychiatry 2012, 57:547-553.

19. Gu L, Xie J, Long J, Chen Q, Chen Q, Pan R, Yan Y, Wu G, Liang B, Tan J, Xie $X$, Wei B, Su L: Epidemiology of major depressive disorder in mainland china: a systematic review. PLoS One 2013, 8:e65356.

20. Bolton P: Cross-cultural validity and reliability testing of a standard psychiatric assessment instrument without a gold standard. J Nerv Ment Dis 2001, 189:238-242.

21. De Jong JTVM, Van Ommeren M: Toward a culture-informed epidemiology: combining qualitative and quantitative research in transcultural contexts. Transcult Psychiatry 2002, 39:422-433.

22. Durieux-Paillard S, Whitaker-Clinch B, Bovier PA, Eytan A: Screening for major depression and posttraumatic stress disorder among asylum seekers: adapting a standardized instrument to the social and cultural context. Can J Psychiatry 2006, 51:587-597.

23. Beck AT, Steer RA: Internal consistencies of the original and revised Beck Depression Inventory. J Clin Psychol 1984, 40:1365-1367.

24. Beck AT, Steer RA, Ball R, Ranieri W: Comparison of Beck Depression Inventories -IA and -II in psychiatric outpatients. J Pers Assess 1996, 67:588-597.

25. Zheng Y, Wei L, Lianggue G, Guochen Z, Chenggue W: Applicability of the Chinese beck depression inventory. Compr Psychiatry 1988, 29:484-489.

26. Schwarzer R, Jerusalem M: Generalized Self-Efficacy Scale. In Meas. Health Psychol. Users Portf. Causal Control Beliefs. Edited by Weinman S, Wright S, Windsor JM. UK: NFER-NELSON; 1995:35-37.

27. Scholz U, Doña BG, Sud S, Schwarzer R: Is general self-efficacy a universal construct? Psychometric findings from 25 countries. Eur J Psychol Assess 2002, 18:242-251.

28. Spielberger CD: Manual for the State-Trait Anger Expression Inventory (STAXI). Odessa, FL: Psychological Assessment Resources; 1988.

29. Maxwell JP, Sukhodolsky DG, Sit CHP: Preliminary validation of a Chinese version of the state-trait anger expression inventory-2. Asian J Soc Psychol 2009, 12:1-11.

30. straus MA: Measuring intrafamily conflict and violence: the conflict tactics (CT) scales. J Marriage Fam 1979, 41:75-88.

31. Weissman MM, Prusoff BA, Thompson WD, Harding PS, Myers JK: Social adjustment by self-report in a community sample and in psychiatric outpatients. J Nerv Ment Dis 1978, 166:317-326.

32. Ferrans CE, Powers MJ: Psychometric assessment of the Quality of Life Index. Res Nurs Health 1992, 15:29-38.

33. Iverson KM, Gradus JL, Resick PA, Suvak MK, Smith KF, Monson CM: Cognitive-behavioral therapy for PTSD and depression symptoms reduces risk for future intimate partner violence among interpersonal trauma survivors. J Consult Clin Psychol 2011, 79:193-202.

34. Ozer EJ, Best SR, Lipsey TL, Weiss DS: Predictors of posttraumatic stress disorder and symptoms in adults: a meta-analysis. Psychol Bull 2003, 129:52-73. 
35. Clapp JD, Beck JG: Understanding the relationship between PTSD and social support: The role of negative network orientation. Behav Res Ther 2009, 47:237-244.

36. Robinaugh DJ, Marques L, Traeger LN, Marks EH, Sung SC, Gayle Beck J, Pollack MH, Simon NM: Understanding the relationship of perceived social support to post-trauma cognitions and posttraumatic stress disorder. J Anxiety Disord 2011, 25:1072-1078.

37. Meyer-Rath G, Over M: HIV Treatment as prevention: modelling the cost of antiretroviral treatment-state of the art and future directions. PLoS Med 2012, 9:e1001247.

38. Fitzpatrick C, Floyd K: A systematic review of the cost and cost effectiveness of treatment for multidrug-resistant tuberculosis. Pharmacoeconomics 2012, 30:63-80.

39. Pooran A, Pieterson E, Davids M, Theron G, Dheda K: What is the cost of diagnosis and management of drug resistant tuberculosis in South Africa? PLoS One 2013, 8:e54587.

40. Cheng Z, Ma N, Yang L, Agho K, Stevens G, Raphael B, Cui L, Liu Y, Yan B, Ma H, Yu X: Depression and Posttraumatic Stress Disorder in Temporary Settlement Residents 1 Year After the Sichuan Earthquake. Asia Pac J Public Health 2013,

41. Neuner F, Schauer M, Klaschik C, Karunakara U, Elbert T: A comparison of narrative exposure therapy, supportive counseling, and psychoeducation for treating posttraumatic stress disorder in an african refugee settlement. J Consult Clin Psychol 2004, 72:579-587.

42. Neuner F, Onyut PL, Ertl V, Odenwald M, Schauer E, Elbert T: Treatment of posttraumatic stress disorder by trained lay counselors in an African refugee settlement: a randomized controlled trial. J Consult Clin Psychol 2008, 76:686-694.

43. Galvanizing mental health research in low- and middle- income countries: the role of scientific journals. Am J Orthopsychiatry 2004, 74:389-392.

44. Wiley-Exley E: Evaluations of community mental health care in low- and middle-income countries: a 10-year review of the literature. Soc Sci Med 2007, 64:1231-1241.

45. Saxena S, Thornicroft G, Knapp M, Whiteford H: Resources for mental health: scarcity, inequity, and inefficiency. Lancet 2007, 370:878-889.

doi:10.1186/1752-1505-8-14

Cite this article as: Jiang et al: Interpersonal psychotherapy versus treatment as usual for PTSD and depression among Sichuan earthquake survivors: a randomized clinical trial. Conflict and Health 2014 8:14.

\section{Submit your next manuscript to BioMed Central and take full advantage of:}

- Convenient online submission

- Thorough peer review

- No space constraints or color figure charges

- Immediate publication on acceptance

- Inclusion in PubMed, CAS, Scopus and Google Scholar

- Research which is freely available for redistribution

Submit your manuscript at www.biomedcentral.com/submit
(O) Biomed Central 